\title{
Psychosocial aspects of the functional gastrointestinal disorders
}

\author{
D A Drossman, F H Creed, K W Olden, J Svedlund, B B Toner, W E Whitehead
}

Chair, Committee on Psychosocial Factors of the Functional

Gastrointestinal Disorders,

Multinational Working

Teams to Develop

Diagnostic Criteria for

Functional

Gastrointestinal

Disorders (Rome II),

University of North

Carolina,

Chapel Hill, NC, USA

D A Drossman

Co-Chair, Committee on Psychosocial

Factors of the

Functional

Gastrointestinal

Disorders,

Multinational Working

Teams to Develop

Diagnostic Criteria for

Functional

Gastrointestinal

Disorders (Rome II),

University of

Manchester,

Manchester, UK

F H Creed

Mayo Clinic-

Scottsdale, Scottsdale, AZ, USA

$\mathrm{K}$ W Olden

Sahlgrenska University Hospital, Göteborg,

Sweden

J Svedlund

Centre for Addiction and Mental Health,

University of Toronto, Ontario, Canada

B B Toner

Professor of Medicine, University of North

Carolina, Chapel Hill,

NC, USA

W E Whitehead

Correspondence to:

Douglas A Drossman, MD,

Division of Digestive

Diseases, 726

Burnett-Womack Bldg, CB

\#7080, University of North

Carolina, Chapel Hill, NC

27599-7080, USA

Email: drossman@

med.unc.edu

\begin{abstract}
The functional gastrointestinal disorders (FGID) are the most frequent conditions seen in gastroenterology practice and comprise a major portion of primary care. Psychosocial factors are important in these disorders with regard to: (1) their effects on gut physiology; (2) their modulation of the symptom experience; (3) their influence on illness behavior; (4) their impact on outcome; and (5) the choice of the therapeutic approach. This paper provides a review and consensus of the existing literature by gastroenterologists, psychiatrists, psychologists, physiologists, and health services investigators. Evidence is provided to support the biopsychosocial model as a basis for understanding and treating these disorders, and epidemiological and clinical information on the relations of psychosocial factors to gut physiology, symptom presentation, health behavior, and outcome is offered. Features of motility, personality, abuse history, health concerns, and treatment-seeking differ between patients with FGID and healthy controls, but they are not specific to FGID. They occur in other patients with chronic medical conditions and/or psychiatric disorders. Review of treatment trials indicates clear support for psychotherapeutic treatments, especially in the long term, as well as some evidence for the benefit of antidepressants in FGID, even in the absence of improvements in mood. (Gut 1999;45(Suppl II):II25-II30)
\end{abstract}

Keywords: functional gastrointestinal disorders; psychologic assessment; psychiatric diagnosis; psychosocial factors; health-related quality of life; psychological treatment; psychopharmacological treatment; Rome II

\section{Introduction}

The functional gastrointestinal disorders (FGID) comprise a major portion of gastrointestinal practice and primary care, and are associated with significant absenteeism from work, impaired health-related quality of life, and increased medical costs. ${ }^{1}$ Psychosocial factors influence gut physiology, the symptom experience, health behavior, and outcome. They are especially important in patients with refractory symptoms - those who are over-represented at referral (tertiary) medical centers.

Although the gut is physiologically responsive to emotional and environmental (stressful) stimuli, there is no consistent psychosocial abnormality associated with these disorders.
Recently, research has shifted from etiologythat is, a unidirectional relationship between psychosocial events (e.g., "stress") and gastrointestinal function, to the reciprocal interaction of physiologic and psychosocial processes in these conditions (the brain-gut axis) (fig 1).

The reader is referred to the physiology and basic science sections of the supplement for additional information.

\section{Brain-gut interactions}

A unifying hypothesis to explain the FGIDs is that they result from dysregulation of brain-gut neuroenteric systems, much like anovulatory bleeding is a dysregulation of hypothalamicpituitary-ovarian function rather than a disease of these structures. The brain-gut neurotransmitters associated with these symptoms are not site specific; they have varied influences on gastrointestinal, endocrine and immune function, and human behavior.

The role of the central nervous system (CNS) in modulating motility is supported by evidence that: (1) the motility disturbances in irritable bowel syndrome (IBS) disappear during sleep ${ }^{2} ;(2)$ the frequency of the migrating motor complex (MMC) decreases and propagating velocity increases progressively with alertness and arousal'; (3) patients with IBS have a different electroencephalography sleep pattern than healthy subjects; and (4) positron emission tomography (PET) studies suggest that the CNS response to rectal distension is altered in patients with IBS compared with controls.

The varied influences of environmental stress, thought, and emotions on gut function help explain the variation in symptoms of patients with these disorders. It also helps explain how psychosocial trauma (e.g., history of physical or sexual abuse ${ }^{3}$ ) or poor coping style (e.g., "catastrophizing") profoundly affects symptom severity, daily function, and health outcome. It is no longer rational to try to discriminate whether physiological or psychologic factors cause pain or other bowel symptoms. Both are operative, and the task is to determine the degree to which each contributes and is remediable.

Abbreviations used in this paper: FGID, functional gastrointestinal disorders; CNS, central nervous system; IBS; irritable bowel syndrome; MMC, migrating motor complex; PET, positron emission tomography; HRQOL, health-related quality of life; CBT, cognitive-behavioral therapy; SSRI, selective serotonin reuptake inhibitor; TCA, tricyclic antidepressant. 
FGID-Conceptual model

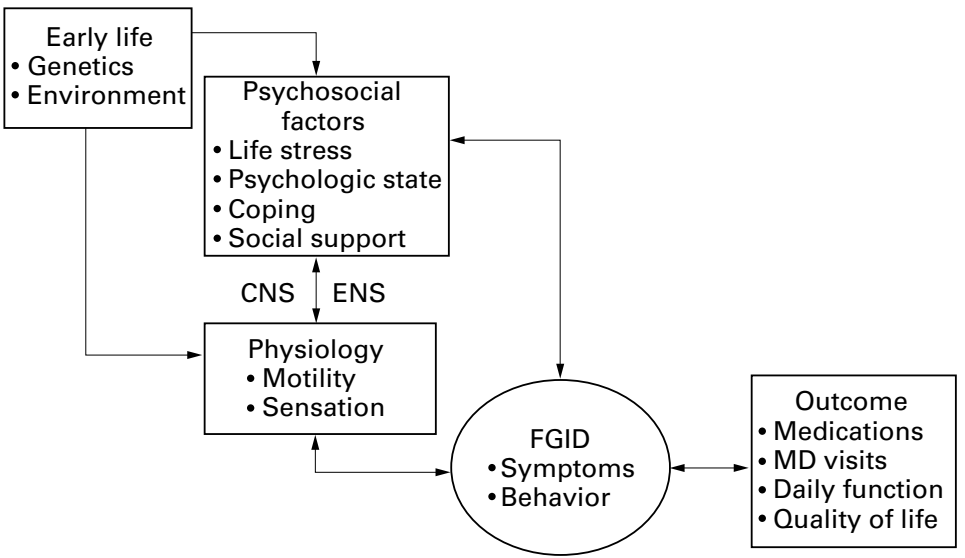

Figure 1 A biopsychosocial conceptualization of the pathogenesis and clinical expression of the functional gastrointestinal disorders (FGID). CNS, central nervous system; ENS, enteric nervous system. problems or the non-clinical population with similar gastrointestinal complaints. ${ }^{7}$ However, there is no personality profile unique to IBS.

HEALTH CARE SEEKING

People with IBS seeking health care, ${ }^{8} 9$ particularly at specialty clinics, have more severe medical symptoms and more anxiety, depression $^{81011}$ and health anxiety, ${ }^{8}$ and they are less likely to see a link between stress and their IBS symptoms. ${ }^{8} 1213$

HEALTH BELIEFS AND ILLNESS BEHAVIORS

Patients with more severe IBS commonly believe that their bowel symptoms indicate serious gut disease/cancer, and attend selectively to abdominal sensations, dismissing other information (e.g., from a doctor) that contradicts such beliefs.

Patients with IBS report many nongastrointestinal disorders. They make two to three times as many visits to physicians for non-gastrointestinal complaints ${ }^{1}$ and report missing an average of 13.4 days from work or usual activities due to illness compared with 4.9 days for the whole sample. ${ }^{1}$ These patterns of somatization and of health care seeking behavior may be learned during childhood. ${ }^{14}$

HEALTH-RELATED QUALITY OF LIFE

Health-related quality of life (HRQOL) refers to the impact of the illness on activities of daily living, the perceptions of the illness, and its consequences. Generic HRQOL instruments are applicable to all medical disorders and indicate that patients with IBS and functional dyspepsia ${ }^{15}$ have impaired quality of life, which is more severe than the impairment in patients with structural abnormalities such as peptic ulcer and liver disease. ${ }^{3}$ Disease-specific instruments to assess quality of life in IBS are now available. ${ }^{1617}$ of abuse history are seen with other chronic pain conditions, a history of abuse is not etiologic for FGID but is associated with a tendency to communicate psychological distress through physical symptoms. ${ }^{6}$ It may also lower gastrointestinal symptom threshold or increase intestinal motility and modify the appraisal of bodily symptoms (i.e., increase medical helpseeking) due to altered cognitions (e.g., feeling ineffective and unable to control the symptoms).

PSYCHIATRIC DISORDERS

For IBS, the most frequent comorbid psychiatric disorders seen include: (1) anxiety disorders (panic and generalized anxiety disorder); (2) mood disorders (major depression and dysthymic disorder); and (3) somatoform disorders (hypochondriasis and somatization disorder).

These are present in between 42 and $61 \%$ of patients seen in gastroenterology clinics compared with $25 \%$ in the control groups. Like abuse history, psychiatric disorder is greater among patients with IBS seen in referral centers than those seen in community clinics.

PERSONALITY

Like patients with other medical disorders patients with IBS have higher trait anxiety and neuroticism scores than people without health

\section{Assessment} desirable for all patients, and is especially important for patients who are refractory to first line medical therapy. Taking a psychosocial history may help to reduce return visits. ${ }^{18}$

\section{OBTAINING THE HISTORY}

The patient is encouraged to relate the history in his or her own way so that the psychosocial events contributing to the illness unfold naturally. ${ }^{19-21}$ The questions should communicate the physician's willingness to address both biologic and psychologic aspects of the illness. A patient-centered style is recommended by maintaining eye contact, not interrupting the patient, and adopting a low control style.

\section{EVALUATING THE ROLE FOR PSYCHOSOCIAL} FACTORS

A few questions can help the physician understand the role of psychosocial factors in a patient's illness ${ }^{192}$ : is the illness acute or chronic; why is the patient coming now; what psychosocial factors are influencing health care seeking; is there a history of unresolved major loss or trauma ${ }^{6}$; does the patient exhibit
A biopsychosocial approach to assessment is 
abnormal illness behavior such as disability disproportionate to observed disease, placement of undue responsibility with the physician, a desire to be taken care of by others, and behaviors that help sustain the patient as an ill person; what is the impact of the illness; is there a psychiatric diagnosis; how does the family interact around the illness?

\section{MEDICAL TESTS}

The use of symptom-based diagnostic criteria (e.g., Rome criteria; see other articles in this supplement) can help to establish a positive diagnosis and minimize unneeded studies. Tests should be based on objective data (e.g., blood in stool, abnormal blood studies, etc.), rather than the patient's insistence to "do something." The physician should also consider safety, whether the results would make a difference to treatment, and whether the test is cost effective.

EVALUATING FOR A PSYCHOLOGIC DISORDER

The physician should screen for anxiety and depression using a few key questions: have you been worrying, had difficulty relaxing, had difficulty with sleep; have you felt low in energy, losing interest and confidence in yourself, and unable to concentrate? If the answers to any of these questions are positive, further psychologic evaluation is needed. In everyday clinical practice, the gastroenterologist should be able to make the diagnoses of depression, panic, agoraphobia, and somatoform disorders; recent diagnostic criteria are available. ${ }^{22}$

More formalized psychological testing usually for research purposes can be performed for the various psychological domains. Some tests require evaluation by a mental health professional (e.g., SCID, HAD, DIS), whereas others (e.g. STAT, BDI) can be self-administered. Categories of tests available include:

(1) Structured psychiatric interviews-for example, using the diagnostic interview schedule (DIS), the structured clinical interview for DSM-IV (SCID), the schedules for clinical assessment in neuropsychiatry (SCAN) which provide DSM-IV, and ICD-10 diagnoses.

(2) Generic psychologic state self-rating scales can be assessed using the symptom checklist-90 (SCL-90) and the general health questionnaire (GHQ).

(3) Syndrome specific self-administered or interview-directed psychologic state questionnaires can be used to assess anxiety or depression. Examples are the Spielberger state trait anxiety inventory (STAI), the Sheehan patient rated anxiety scale, the Beck depression inventory (BDI), and the hospital anxiety and depression (HAD) scale.

(4) Personality is measured with selfadministered questionnaires including the MMPI, ${ }^{23}$ the Eysenck personality inventory (EPI) and the neuroticism, extroversion, openness score (NEO).

(5) Illness behaviors and attitudes are measured using the illness behavior questionnaire or the illness attitude scale. ${ }^{24}$
(6) Cognitive scales are designed to measure aspects of attitudes towards illness. The cognitive scale for functional bowel disorders has recently been developed for use as an assessment and outcome measure for FGIDs. ${ }^{25}$

(7) Social support is only reliably measured by an interview. Self-report questionnaires indicate perceived quantity and quality of the social support (e.g., Sarason social support questionnaire $\left.(\mathrm{SSQ})^{26}\right)$.

(8) Coping is evaluated using the ways of coping questionnaire, the coping strategies questionnaire (CSQ) and its catastrophizing scale, which measures maladaptive coping strategies that predict adverse health outcome among patients with gastrointestinal disorders.

(9) Health-related quality of life can be evaluated using generic HRQOL instruments (e.g., short form $(\mathrm{SF}-36))^{27}$ and disease-specific HRQOL instruments (e.g., irritable bowel syndrome quality of life instrument (IBS-QOL)) ${ }^{17}$ and irritable bowel syndrome quality of life questionnaire (IBSQOL). ${ }^{16}$

(10) Further details of these measures are found in the full publication. ${ }^{5}$

\section{Treatment}

\section{APPROACH TO THE PATIENT}

Therapeutic relationship

This can be developed when the physician: (1) elicits and acknowledges the patient's beliefs, concerns, and expectations; (2) offers empathy when needed; (3) clarifies misunderstandings; (4) provides education; and (5) negotiates a plan of treatment with the patient. ${ }^{28}$ Some patients are initially unwilling to accept a role for psychosocial factors in the illness; this difficulty is commonly seen in patients who have suffered severe developmental trauma such as sexual abuse.

\section{Associating bowel symptoms with psychosocial factors}

Assessment of a link between bowel symptoms and psychosocial factors is aided by a daily record of the symptoms along with the time of bowel movements and the timing of menstruation, which can be compared with dietary, lifestyle changes, or stressors. This information can provide the basis for cognitive-behavioral strategies.

\section{Reassurance}

Reassurance can only be achieved after the physician fully understands the patient's particular concerns. The overall favorable prognosis for the FGIDs can then be stressed.

\section{Accept the adaptations of chronic illness}

When the symptoms are chronic, the patient should be helped to reconceptualize the nature of the illness as a set of troublesome symptoms rather than an indication of underlying pathology. In some patients, chronic illness may provide attention from others, release from usual responsibilities, and social and 
financial compensation. In these situations, clinical improvement may take a long time, but may be advanced if the physician focuses more on improving the patient's function in the presence of illness rather than attempting to "cure." The physician must minimize diagnostic studies and symptomatic treatments and work toward reinforcing health-promoting behavior in the patient.

Referral to a mental health professional

Problems which might require referral for consultation and treatment include: (1) psychiatric disorders (e.g., major depression, panic disorder) which require specific treatments (e.g., antidepressants, cognitive-behavioral therapy (CBT) or other psychotherapy); (2) a history of abuse which comes to light during consultation and may be interfering with adjustment to the current illness; (3) serious impairment in daily function which requires specific treatment to improve coping skills; and (4) somatization, where multiple symptoms are leading to numerous consultations across specialties. In order for the gastroenterologist to refer a patient to a mental health professional, he or she must acknowledge the relevance of the psychosocial aspects to the patient's presenting problem. Otherwise the patient may resist the referral because of perceived stigma or a rejection ("the workup is negative, it must be nerves") by the gastroenterologist. Continued care by the gastroenterologist may also be necessary.

\section{The psychotherapies}

While the mental health consultant can select from different types of psychological treatments (e.g., CBT, dynamic psychotherapy, hypnotherapy, and relaxation), experience and the empirical research suggests that no one treatment is superior for FGIDs. The most important aspect of treatment is the patient's acceptance of the need for treatment and his/her motivation to engage in it. This can be enhanced if the gastroenterologist and psychologist/psychiatrist help the patient accept the treatment as a necessary part of an overall plan of care.

Cognitive-behavioral therapy consists of a wide range of strategies and procedures designed to bring about alterations in patients' perceptions of their situation and their ability to control their gastrointestinal symptoms by learning new ways of thinking and behaving through personal experience and practice. In addition, the benefit of this type of treatment is supported by: (a) a high prevalence of anxiety, depression, and assertion difficulties; $(b)$ a high need for social approval; and (c) perfectionistic attitudes; all of these are amenable to CBT. ${ }^{29}$

Dynamic psychotherapy is similar to brief interpersonal psychotherapy ${ }^{30}$ and requires a close relationship between the patient and therapist, in which difficulties in interpersonal relationships can be highlighted. As the patient understands these problems, he or she may act upon these insights, which may lead to a reduction in symptoms.
Hypnotherapy can be applied. The hypnotic state is a state of heightened suggestibility in which the hypnotherapist uses progressive muscular relaxation plus suggestions of relaxation to reduce striated muscle tension and to relax gastrointestinal smooth muscle. Patients are also asked to practice autohypnosis at home with an audiotape with the goal of being able to self-administer suggestions of relaxation. ${ }^{31}$

Relaxation (arousal reduction) training includes a variety of different methods to teach patients to counteract the physiological sequellae of stress or anxiety. The most widely used arousal reduction techniques include: (1) progressive muscle relaxation training; (2) biofeedback for striated muscle tension, skin temperature, or electrodermal activity; (3) autogenic training; and (4) transcendental or Yoga meditation.

EMPIRICAL SUPPORT FOR PSYCHOTHERAPIES

Most of the research to date has focused on IBS and has involved various combinations of cognitive-behavioral, relaxation, psychodynamic, and biofeedback approaches, making assessment of the effectiveness of the specific approaches difficult. Furthermore, patient selection also differs across studies. ${ }^{30}$

We reviewed 15 studies that used a controlled design to compare psychological treatment with conventional medical treatment disorders. ${ }^{5}$ There is an inadequate number of well-designed studies to perform a metaanalysis. We excluded two studies with a participation rate of less than $40 \%$. In terms of reduction of bowel symptoms at the end of treatment, 10 of 13 studies showed significant superiority of psychological over conventional medical treatment. Of the nine studies with follow up data (duration 9-40 months), eight showed superiority of psychological treatment. Only six studies also controlled for expectancy and time with therapist, five of which showed a significantly greater improvement in bowel symptoms in the psychological treatment groups.

Therefore, psychological treatment appears superior to conventional medical treatment and there were no differences in outcome based on technique. The psychotherapist should use the technique with which they are most experienced.

\section{Psychopharmacology}

The rationale for using psychotropic agents lies in the high comorbidity: roughly half of the patients with a FGID also have depression and/or anxiety disorders, which may respond to psychopharmacological intervention. In addition, data support the efficacy of antidepressants in the relief of chronic pain. Data supporting the efficacy of antidepressants in FGID is growing. At least five studies have shown efficacy that is independent of change in mood. ${ }^{5}$

Prescribing psychopharmacologic agents is best accomplished in the context of a strong doctor-patient relationship, where these agents are complementary to an overall multicomponent treatment plan. The physician needs to 
explain the rationale, possible side effects, and expected benefits from the medication and address them in the context of the patient's beliefs and expectations relative to psychopharmacologic treatment.

There is insufficient evidence to recommend one particular type of antidepressant. The choice therefore depends on the target symptoms, the overall clinical picture, and the possible side effects. The response to antidepressant therapy is highly patient specific, namely the side effects and therapeutic effects vary across individuals, making sensible change of drugs appropriate. However, it is better to ensure consistent treatment at an appropriate dose level over a longer period of time (2-3 months) than change rapidly from one drug to another.

The patient with abdominal pain, diarrhea, or nausea would probably do less well with a selective serotonin reuptake inhibitor (SSRI) than on a tricyclic antidepressant (TCA), because the SSRIs produce cramping, nausea, and diarrhea due to their prokinetic effect. There is evidence from a meta-analysis that a variety of pain conditions shows superior response to TCAs than SSRIs. SSRIs may lead to side effects in the gastrointestinal tract, though these generally settle with continued treatment. A patient with considerable anxiety might do better on an antidepressant that tends to be more sedating - that is, one with a strong antihistaminic effect.

The SSRIs have not been well studied in the FGIDs. However, the SSRIs have a number of qualities which make them potentially useful in certain gastrointestinal settings. The prokinetic effects of SSRIs may make them particularly helpful in patients who have functional constipation and/or functional abdominal bloating. In addition, recent studies supporting a role for central 5-hydroxytryptamine dysfunction in non-ulcer dyspepsia may suggest a use for these agents. ${ }^{5}$ The usefulness of other antidepressants has not yet been established in the FGIDs, but they provide an alternative for patients who cannot tolerate SSRIs because of side effects.

Anxiolytic agents are effective for reducing anxiety in the short term, but their CNS depressant effect, including mild transient cognitive dysfunction and the risk of addiction with the benzodiazepines, leads us to recommend that a psychiatrist be consulted to evaluate patients before prescribing benzodiazepines on a long term basis. Alternative strategies for the treatment of anxiety should be used.

\section{Recommendations for future research}

This review has indicated the importance of psychosocial variables in FGID but further research is required: (1) studies are needed that will standardize current measures and develop new instruments for FGID to examine the interaction between psychosocial traits (e.g., neuroticism and HRQOL) and bowel symptoms; (2) psychological intervention studies should include session-by-session treatment manuals and measures of therapist adherence to treatment protocols and use different appropriate placebo conditions to address expect- ancy and attention, and measure credibility; (3) well-designed, randomized, controlled psychopharmacologic trials for psychotropic drugs are needed.

The influence of gender and sociocultural factors and the influence of clinical setting (non-patients, primary care, gastrointestinal referral, psychiatric referral) need to be more fully understood. New conceptual models for the pathogenesis of FGID are needed, and prospective studies to assess populations at risk will be helpful (e.g., children of parents with FGID, those who acquire enteric infections, victims of abuse). The role of genetic factors on the presence of FGID and the selectivity to specific organ systems should be studied further. Future studies that combine brain imaging (PET, functional magnetic resonance imaging), gastrointestinal physiology, and standardized psychosocial assessment to determine whether treatment effects (pharmacological and psychological) on symptoms mediated by changes in gut/CNS physiology.

Treatment studies will help us to understand the effect of physician communication skills on physician/patient satisfaction with care, adherence to treatment including drug trial and outcome. Further studies are required to determine the patient characteristics which predict response to specific psychological treatments and which components of psychological treatment packages (e.g., relaxation, cognitive restructuring, etc.) account for their effectiveness. Treatment effects in the long term need to be studied, and there have been no studies to date assessing the appropriate duration of the psychological and psychopharmacological treatments. We would like to thank Drs Susan Levenstein, Rona Levy, Claus
Buddeberg, Nick Diamant, Marvin Schuster, Gabriele Moser for their critical review of this manuscript, and Ms Carlar for their critical review of this manuscript, and Ms Carlar preparation of this document.

1 Drossman DA, Li Z, Andruzzi E, et al. U.S. householder survey of functional gastrointestinal disorders. Prevalence, sociodemography and health impact. Dig Dis Sci 1993;38:1569-80

2 Kellow JE, Gill RC, Wingate DL. Prolonged ambulant recordings of small bowel motility demonstrate abnormalities in the irritable bowel syndrome. Gastroenterology 1990; 98:1208-18.

3 Drossman DA, Li Z, Leserman J, et al. Health status by gastrointestinal diagnosis and abuse history. Gastroenterology 1996;110:999-1007.

4 Creed FH, Craig T, Farmer RG. Functional abdominal pain, psychiatric illness and life events. Gut 1988;29:23542 .

5 Drossman DA, Creed FH, Olden KW, et al. Psychosocial aspects of the functional gastrointestinal disorders. In: Drossman DA, Talley NJ, Thompson WG, Corazziari E, Whitehead WE, eds. Rome II: The functional gastrointestinal disorders: Diagnosis, pathophysiology and treatment; A multinadisorders: Diagnosis, pathophysiology and treatment; A multina-

tronal consensus. McLean, VA: Degnon and Associates,
6 Drossman DA, Talley NJ, Olden KW, et al. Sexual and
6 . Drossman DA, Talley NJ, Olden KW, et al. Sexual and
physical abuse and gastrointestinal illness. Review and recommendations. Ann Intern Med 1995;123:782-94.

7 Drossman DA, Whitehead WE, Camilleri M. Irritable bowel syndrome. A technical review for practice guideline development. Gastroenterology 1997;112:2120-37.

8 Drossman DA, McKee DC, Sandler RS, et al. Psychosocial factors in the irritable bowel syndrome. A multivariate study of patients and nonpatients with irritable bowel syndrome. Gastroenterology 1988;95:701-8.

9 Whitehead WE, Bosmajian L, Zonderman AB, et al. Symptoms of psychologic distress associated with irritable bowel syndrome. Comparison of community and medical clinic syndrome. Comparison of community and
samples. Gastroenterology 1988;95:709-14.

10 Heaton KW, O'Donnell LJD, Braddon FEM, et al. Symptoms of irritable bowel syndrome in a British urban community: consulters and nonconsulters. Gastroenterology 1992;102:1962-7. 
11 Talley NJ, Boyce PM, Jones M. Predictors of health care seeking for irritable bowel syndrome: a population based study. Gut 1997;41:394-8.

12 Drossman DA, Sandler RS, McKee DC, et al. Bowel patterns among subjects not seeking health care: use of a questionnaire to identify a population with bowel dysfunction. Gastroenterology 1982;83:529-34.

13 Toner BB, Segal ZV, Emmott S, et al. Cognitive-behavioral group therapy for patients with irritable bowel syndrome. Int f Group Psychother 1998;48:215-43.

14 Whitehead WE, Crowell MD, Heller BR, et al. Modeling and reinforcement of the sick role during childhood predicts adult illness behavior. Psychosomat Med 1994;6: $541-50$

15 Talley NJ, Weaver AL, Zinsmeister AR. Impact of functional dyspepsia on quality of life. Dig Dis Sci 1995;40:84-9.

16 Hahn BA, Kirchdoerfer LJ, Fullerton S, et al. Evaluation of a new quality of life questionnaire for patients with irritable bowel syndrome. Aliment Pharmacol Ther 1997;11: 547-52.

17 Patrick DL, Drossman DA, Frederick IO, et al. Quality of life in persons with irritable bowel syndrome. Development life in persons with irritable bowel syndrome. Deve.

18 Owens DM, Nelson DK, Talley NJ. The irritable bowel syndrome. Long term prognosis and the physician-patient interaction. Ann Intern Med 1995;122:107-12.

19 Drossman DA. Psychosocial factors in the care of patients with gastrointestinal disorders. In: Yamada T, ed. Textbook of gastroenterology. Philadelphia: Lippincott-Raven, 1999 (in press).

20 Drossman DA. Struggling with the "controlling" patient. Am $\mathcal{f}$ Gastroenterol 1994;89:1441-6.
21 Olden KW. Approach to the patient with irritable bowel syndrome. In: Stern TA, Herman JB, Slavin PL, eds. The
MGH guide to psychiatry in primary care. New York: MGH guide to psychiatry

22 American Psychiatric Association. Diagnostic and statistical manual of mental disorders-DSM-IV. Washington, DC: American Psychiatric Association, 1994.

23 Dahlstrom WG, Welsh GS, Dahlstrom LE. An MMPI handbook. Revised edition. Minneapolis: University of Minnesota, 1972.

24 Kellner R. Somatization and hypochondriasis. New York: Prager-Greenwood, 1986.

25 Toner BB, Stuckless N, Ali A, et al. The development of a cognitive scale for functional bowel disorders. Psychosom Med 1998;60:492-7.

26 Sarason IG, Sarason BR, Shearin EN, et al. A brief measure of social support. Practical and theoretical implications. .

27 Ware JE, Sherbourne CD. The MOS 36-item short form Health Survey (SF-36). I. Conceptual framework and item selection. Med Care 1992;30:473-83.

28 Drossman DA. Psychosocial sound bites. Exercises in the patient-doctor relationship. Am f Gastroenterol 1997;92: patient-d $1418-23$

29 Toner BB. Cognitive-behavioral treatment of functional somatic syndromes: integrating gender issues. Cognitive and Behavioral Practice 1994;1:157-78.

30 Guthrie E, Creed F, Dawson D, et al. A randomised controlled trial of psychotherapy in patients with refractory irritable bowel syndrome. Br f P Pychiatry 1993;163: 315-21.

31 Whorwell PJ. Use of hypnotherapy in gastrointestinal disease. Br f Hosp Med 1991;45:27-9.

\section{For further information and updates on Rome II, visit our website at:

